Published in final edited form as:

Gastroenterology. 2008 July ; 135(1): e5-e6. doi:10.1053/j.gastro.2008.06.018.

\title{
Electronic Clinical Challenges and Images in GI
}

ERIK C. VON ROSENVINGE, MD, CHRISTOPHER KOH, MD, YARON ROTMAN, MD, and RAMONA M. LIM, MD

National Institute of Diabetes and Digestive and Kidney Diseases National Institutes of Health Bethesda, Maryland

Question. A 44-year-old man with a history of hepatitis C was enrolled in a treatment protocol with pegylated interferon and ribavirin. Three months into therapy, the patient experienced several self-limited episodes of garbled speech and right thigh numbness. The patient was otherwise asymptomatic. Neurologic examination was significant only for mildly impaired tandem gait. Physical examination was otherwise normal, including no stigmata of chronic liver disease and no significant abdominal, chest, skin, or lymphatic findings.

Non-contrast-enhanced computed tomography (CT) of the head and magnetic resonance imaging (MRI) of the brain revealed multiple intraparenchymal hemorrhages of unclear etiology. Further evaluation with contrast-enhanced CT of the chest, abdomen, and pelvis revealed mediastinal and right hilar lymphadenopathy, questionable thickening of the gastric fundus, a $2.8-\mathrm{cm}$ ulcerated mass in the distal duodenum (Figure A), and a $3.2-\mathrm{cm}$ mass versus intussusception in the ileum. Enteroscopy was performed and 2 adjacent $2.0-\mathrm{cm}$ ulcerated masses were found in the gastric fundus (Figure B) and biopsied. A similar distal duodenal lesion was identified and corresponded with the CT findings. What is the diagnosis?

\section{References}

1. Kawakami Y, Battles J, Kobayashi T, et al. Production of recombinant MART-1 proteins and specific antiMART-1 polyclonal and monoclonal antibodies: use in the characterization of the human melanoma antigen MART-1. J Immunol Meth 1997;202:13-25.

2. Krüger S, Noack F, Blöchle C, et al. Primary malignant melanoma of the small bowel: a case report and review of the literature. Tumori 2005;91:73-76. [PubMed: 15850009]

3. Schuchter L, Green R, Fraker D. Primary and metastatic diseases in malignant melanoma of the gastrointestinal tract. Curr Opin Oncol 2000;12:181-185. [PubMed: 10750731]

See the GASTROENTEROLOGY web site (www.gastrojournal.org) for more information on submitting your favorite image to Clinical Challenges and Images in GI. 


\section{Answer to the Clinical Challenges and Images in GI Question: Image 3: Gastrointestinal Malignant Melanoma}

Endoscopically, the gastrointestinal masses were ulcerated with violaceous pigmentation typical of malignant melanoma. Histologic evaluation of biopsy specimens from the fundic and duodenal lesions revealed sheets of pigmented malignant epithelioid cells with pink cytoplasm and prominent nucleoli (Figure C; hematoxylin and eosin staining). By immunohistochemistry, the cells were all strongly positive for MART-1 ${ }^{1}$ (Figure D; positive cells shown with red chromagen), S-100, Melan-A, HMB-45, and tyrosinase, confirming the presence of malignant melanoma. The patient underwent formal dermatologic and ophthalmologic evaluations, and no evidence of a cutaneous or ocular primary malignant melanoma was found.

The vast majority of gastrointestinal malignant melanomas are a result of metastatic disease; however, melanocytes are known to be present in the gastrointestinal tract, and there have been prior reports of primary melanomas arising in the esophagus, stomach, small intestine, ${ }^{2}$ colon, and anorectum. Primary malignant melanomas of the gastrointestinal tract have been described as being aggressive and associated with a worse prognosis. ${ }^{3}$ This may be a reflection of their identification at a more advanced stage (as in our patient). Although this patient's disease is suspicious for a primary gastrointestinal malignant melanoma, it is presently impossible to prove that his melanoma originated in the gastrointestinal tract. 


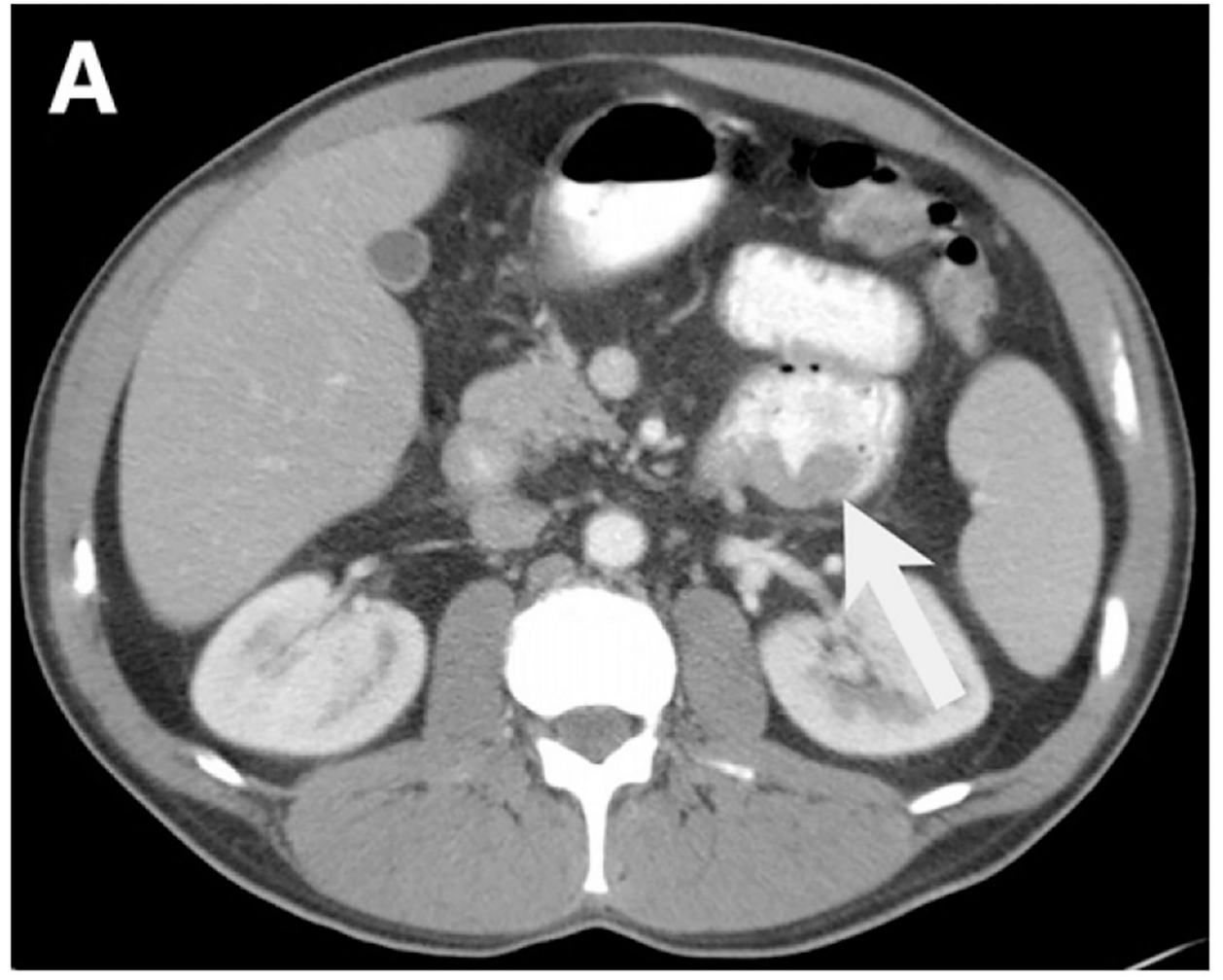

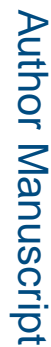

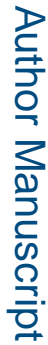




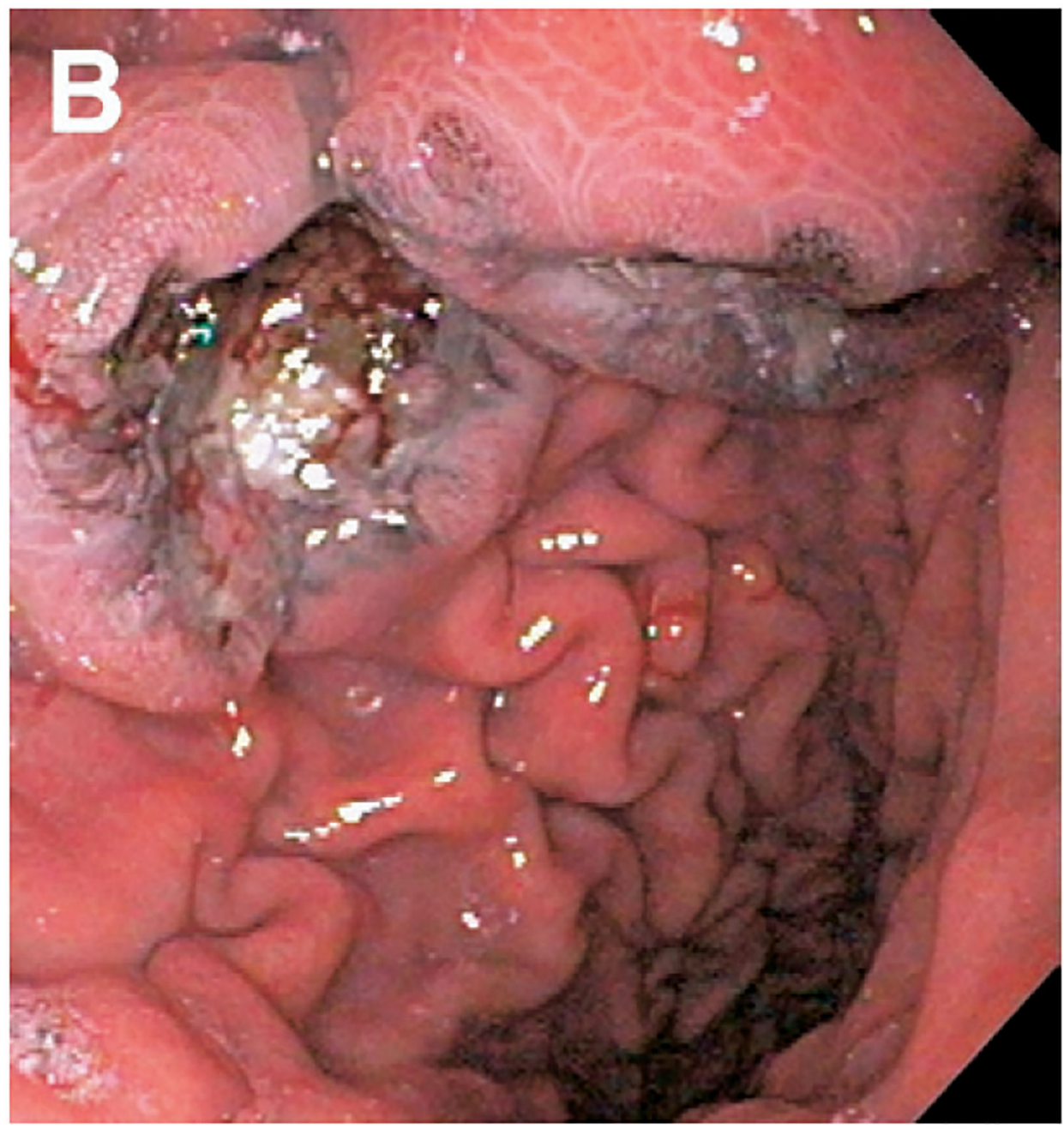

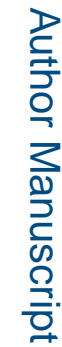



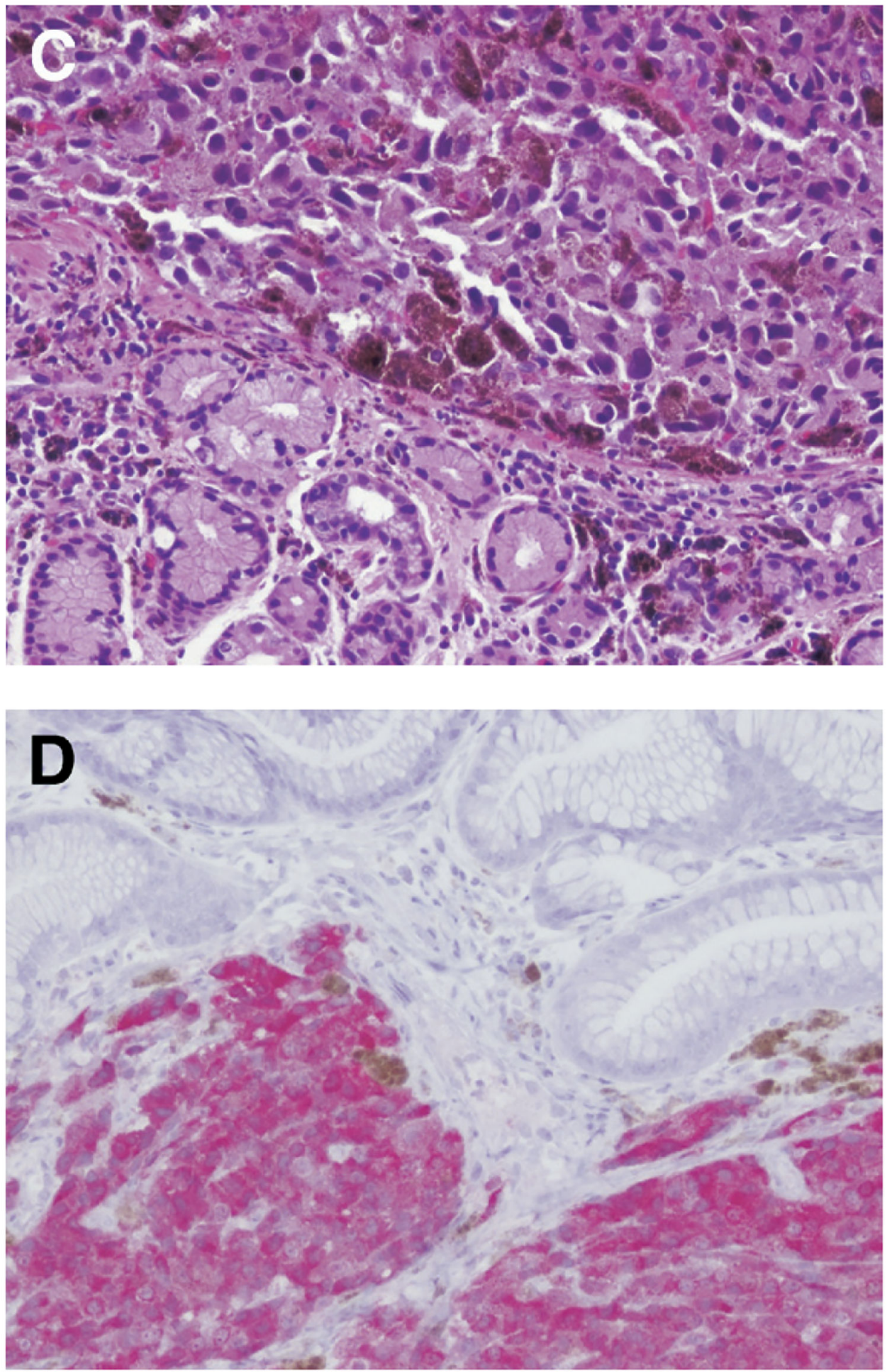

Image 3. 